\title{
TOROIDAL CONSIDERATION OF PLASMA RESPONSE TO PENETRATION OF AN EXTERNAL LOW FREQUENCY HELICAL PERTURBATION INTO A TOKAMAK EDGE PLASMA
}

\author{
I.M. Pankratov, A.Ya. Omelchenko, V.V. Olshansky \\ Institute of Plasma Physics, \\ National Science Center "Kharkov Institute of Physics and Technology", \\ Akademicheskaya str.1, 61108 Kharkov, Ukraine,e-mail: pankratov@kipt.kharkov.ua
}

For an adequate comparison with TEXTOR-DED experiments [1] the theoretical treatment of the DED operation, with the plasma response has been taken into account, are made in the toroidal geometry on the basis of two-fluid MHD PACS: 52.55.Dy; 52.55.Fa; 52.55.Rk

\section{INTRODUCTION}

Control of plasma edge behaviour is the main purpose of the Dynamic Ergodic Divertor (DED) project of TEXTOR [1]. The DED helical coils create a specific topology of magnetic field at the plasma edge, where external rotating helical perturbations are resonant on the rational magnetic surfaces.

In cylindrical model the penetration of external perturbations was investigated in [2]. The toroidal modelling (without consideration of the plasma response) shows a more strong perturbation decrease with the distance from DED coils as compared with the cylindrical model (see, e.g., [1]). In the present paper for an adequate comparison with DED experiments the theoretical treatment of the DED operation is investigated in the toroidal geometry on the basis of two-fluid MHD equations with the plasma response being taken into account.

\section{BASIC EQUATIONS}

We start from the two-fluid MHD equations for continuity, momentum and energy conservation for plasma ions and electrons and the Maxwell's equations. The electron-ion collision frequency is higher than the frequency of external perturbation. The electron inertia and electron stress tensor are neglected. Then by the usual way we take the plasma equation of motion

$$
\rho\left(\frac{\partial \mathbf{V}}{\partial t}+(\mathbf{V} \cdot \nabla) \mathbf{V}\right)=-\nabla p-\nabla \cdot \boldsymbol{\pi}_{i}+[\mathbf{J} \times \mathbf{B}],
$$

and the generalized Ohm's law $(e>0)$

$$
\begin{gathered}
\mathbf{J}_{\|} / \sigma_{\|}+\mathbf{J}_{\perp} / \sigma_{\perp} \\
=\mathbf{E}+[\mathbf{V} \times \mathbf{B}]+\nabla p_{e} / e n_{e}-[\mathbf{J} \times \mathbf{B}] / e n_{e}+(0.71 / e) \nabla_{\|} T_{e},
\end{gathered}
$$

where $n_{e}$ and $\rho$ are the plasma and plasma mass densities, $p=p_{e}+p_{i}$ is the total pressure, $\mathbf{J}$ is the total current density, $\boldsymbol{\pi}_{i}$ is the ion gyroviscosity tensor, $\sigma_{\|}$ and $\sigma_{\perp}$ are the parallel and perpendicular (with respect to the magnetic field $\mathbf{B}$ ) conductivities, respectively. The parallel electron heatconductivity coefficient is assumed to be a large value. Hence, in our approximation ( $T_{e}$ is the electron temperature)

$$
\text { B } \nabla T_{e}=0 \text {. }
$$

We use the approximation of an incompressible plasma motion $\nabla \cdot \mathbf{V}^{\sim}=0$. We consider a current carrying toroidal plasma with nested equilibrium circular magnetic surfaces ( $\rho_{0}$ is the radius of the magnetic surfaces, $\omega_{0}$ is the poloidal angle in the cross-section $\zeta=$ const,$\zeta$ is the toroidal angle). Each magnetic surface is shifted with respect to the magnetic axis ( $\xi$ is the shift, $R$ is the radius of the magnetic axis). The equilibrium toroidal contravariant component of the magnetic field, $B_{0}^{\zeta}=\Phi^{\prime} /(2 \pi \sqrt{g})$, is large with respect to the poloidal one, $B_{0}^{\theta}=\chi^{\prime} /(2 \pi \sqrt{g}), \Phi^{\prime}$ and $\chi^{\prime}$ are the radial derivatives of toroidal and poloidal fluxes, respectively.

By a usual manner (see, e.g., [3]) on each magnetic equilibrium surface we introduce the straight magnetic field line coordinate system $(a, \theta, \zeta)$

$$
\begin{gathered}
\rho_{0}=a, \omega_{0}=\theta+\lambda(a) \sin \theta \\
\lambda(a)=-\xi^{\prime}(a)-a / R \\
\xi^{\prime}(a)=\frac{1}{a R}\left(\frac{\chi^{\prime}(a)}{2 \pi R}\right)^{-2} \int_{0}^{a}\left[4 \mu_{0} p_{0}(b)+\left(\frac{\chi^{\prime}(b)}{2 \pi R}\right)^{2}\right] b d b
\end{gathered}
$$

Equations (4) are valid for a low plasma pressure $p_{0}(a)$ and a small toroidicity $a / R$ when it is possible to neglect the magnetic surfaces deformation. The well-known expressions for metric tensor are used [3].

Assuming periodicity in both $\theta$ and $\zeta$, we take contravariant components of perturbations in the form

$$
X^{a, \theta}(a, \theta, \zeta, t)=\sum_{m, n} X_{m, n}^{a, \theta}(a) \exp [i(m \theta-n \zeta-\omega t)],
$$

where $\omega$ is the frequency of the external perturbation. The toroidal symmetry of the TEXTOR-DED system gives $n=(2 k+1) n_{0},\left(n_{0}=4, k=0,1, \ldots\right)$. Because of the strong radial decay of the $k>1$ harmonics, the perturbations can be approximated in Eq. (7) and below by the toroidal Fourier terms with $n=n_{0}$ (see [4]). For contravariant components of plasma velocity and magnetic field perturbations, $V_{m}^{a, \theta}$ and $B_{m}^{a, \theta}$, the system of equations is obtained in the linear approximation. These equations take into account the resistive effects and 
toroidal modes coupling. Poloidal mode numbers $m$ are defined by the DED antenna spectrum.

$$
\begin{aligned}
& i \omega_{i} B_{m}^{a}=-i F_{m}(a) V_{m}^{a}-\frac{\omega \delta^{2}(\omega)}{a^{2}}\left[\frac{\partial}{\partial a} a \frac{\partial}{\partial a} a B_{m}^{a}-m^{2} B_{m}^{a}\right] \\
& -i \frac{a}{R}\left[F_{m-1}(a) V_{m-1}^{a}+F_{m+1}(a) V_{m+1}^{a}\right]+i \frac{a}{R} V_{0}^{\theta}\left[B_{m-1}^{a}-B_{m+1}^{a}\right]-\frac{\omega \delta^{2}(\omega)}{a^{2}} m^{2} \lambda\left(B_{m-1}^{a}+B_{m+1}^{a}\right), \\
& \frac{d}{d a} a \omega^{\prime} \rho \frac{d}{d a} a V_{m}^{a}-m^{2} \omega^{\prime} \rho V_{m}^{a}=-\frac{F_{m}(a)}{\mu_{0}}\left(\frac{d}{d a} a \frac{d}{d a} a B_{m}^{a}-m^{2} B_{m}^{a}\right)+\frac{a B_{m}^{a}}{\mu_{0}}\left(a \frac{d^{2}}{d a^{2}}+3 \frac{d}{d a}\right)\left(\frac{m}{a} \frac{\chi^{\prime}}{2 \pi R}\right) \\
& +\frac{a}{\mu_{0}} \frac{d a B_{m}^{a}}{d a} \cdot \frac{d}{d a} \frac{n}{R} \frac{\Phi^{\prime}(a)}{2 \pi a}-\frac{\partial}{\partial a} \rho \frac{a}{R} \omega^{\prime}\left[\frac{\partial}{\partial a}\left(\sqrt{g} V^{a}\right)+\frac{\partial}{\partial \theta}\left(\sqrt{g} V^{\theta}\right)\right]_{m} \\
& +i m\left\{\frac{\partial}{\partial a} \rho\left[\omega^{\prime}\left(g_{12} V^{a}+g_{22} V^{\theta}\right)+i V_{0}^{\theta}\left(\dot{g}_{12} V^{a}+\dot{g}_{22} V^{\theta}\right)\right]-\rho \frac{\partial}{\partial \theta}\left[\omega^{\prime}\left(g_{11} V^{a}+g_{12} V^{\theta}\right)+i V_{0}^{\theta}\left(\dot{g}_{11} V^{a}+\dot{g}_{12} V^{\theta}\right)\right]\right\}_{m} \\
& +\frac{\partial}{\partial \theta}\left\{\frac{\partial}{\partial a}\left[\frac{\chi^{\prime}}{2 \pi a R}\left(g_{22}-\frac{a}{R} \sqrt{g}\right) B^{\theta}\right]+\frac{\chi^{\prime}}{2 \pi a R}\left[\left(g_{22}^{\prime}-\frac{2}{R} \sqrt{g}\right) B^{\theta}-\left(g_{11}-\frac{1}{a R} \sqrt{g}\right) \frac{\partial B^{a}}{\partial a}\right]\right\}_{m} \\
& +\frac{\partial}{\partial a}\left\{-i n \frac{\Phi^{\prime}}{2 \pi a R}\left(g_{22}-\frac{a}{R} \sqrt{g}\right) B^{\theta}+B^{a} \frac{\partial}{\partial a}\left[\frac{\chi^{\prime}}{2 \pi a R}\left(g_{22}-\frac{a}{R} \sqrt{g}\right)\right]\right\}_{m}-\frac{\partial}{\partial a}\left[\frac{\chi^{\prime}}{2 \pi a R}\left(i n q(a) g_{12}+\dot{g}_{12}\right) B^{a}\right]_{m} \\
& +\frac{\chi^{\prime}}{2 \pi a R} \frac{\partial}{\partial \theta}\left[-\left(\dot{g}_{12} B^{\theta}+\frac{\partial}{\partial \theta} g_{12} B^{\theta}\right)+\left(g_{12}^{\prime}-\dot{g}_{11}\right) B^{a}+g_{12} \frac{\partial B^{a}}{\partial a}+i n q(a) g_{12} B^{\theta}\right]_{m} .
\end{aligned}
$$

Here

$$
\begin{aligned}
& F_{m}(a)=\frac{1}{2 \pi a R}\left(m \chi^{\prime}(a)-n \Phi^{\prime}(a)\right), \\
& \omega_{i}=\omega-m V_{0}^{\theta}+n V_{0}^{\zeta}, \delta=1 / \sqrt{\mu_{0} \sigma_{\|} \omega} .
\end{aligned}
$$

The value of $F_{m}(a)$ is equal to zero inside the plasma, when $q\left(a_{r e s}\right)=m / n\left(q(a)=\Phi^{\prime} / \chi^{\prime}\right.$ is the safety factor).

Equation (9) contains only oscillating parts of the metric tensor: $g_{11}=2 \xi^{\prime} \cos \theta, \quad g_{12}=a\left(a \lambda^{\prime}-\xi^{\prime}\right) \sin \theta$, $g_{22}=2 a^{2} \lambda \cos \theta, \quad \sqrt{g}=-2 a^{2} \cos \theta, \quad g_{i k}^{\prime}=\partial g_{i k} / \partial a$, $\dot{g}_{i k}=\partial g_{i k} / \partial \theta$, Eq. (9) is written in the compact form: $(f)_{m}$ is the $m$-th poloidal harmonic of $f$, the variables $V^{a, \theta}$ and $B^{a, \theta}$ are the total radial and poloidal perturbations of the magnetic field and plasma velocity, respectively. In Eqs. (8), (9) the small terms that are proportional to $V_{m}^{\zeta}$ and $B_{m}^{\zeta}$ are omitted. We included the equilibrium poloidal plasma rotation with the velocity $V_{0}^{\theta}$ due to existence of an equilibrium radial electric field, here we also took into account the ion diamagnetic drift velocity and the equilibrium toroidal plasma rotation with a homogeneous velocity $V_{0}^{\zeta}$. When we neglect toroidicity, we use physical components of the vectors. For $\omega_{i}$ and $\omega^{\prime}$ we have in this case

$$
\begin{aligned}
& \omega_{i}=\omega^{\prime}-\left(\frac{m}{a}\right)\left(\frac{1}{e n_{e 0} B_{\zeta 0}}\right)\left(\frac{d p_{i 0}}{d a}\right), \\
& \omega^{\prime}=\omega+\left(\frac{m}{a}\right)\left(\frac{E_{a 0}}{B_{\zeta 0}}\right)+k V_{\zeta 0} .
\end{aligned}
$$

The equilibrium current density is included in Eq. (9). We assume that equilibrium quantities are slowly varying. Recall that the ion gyroviscosity tensor $\boldsymbol{\pi}_{i}$ compensates the drift diamagnetic effect [3].

\section{RESULTS AND DISCUSSION}

The first lines of Eqs. (8), (9) in coordinates $(a, \theta)$ formally coincide with Eqs. (4), (7) of cylindrical model [2]. Recall that the solutions of cylindrical equations are shown for TEXTOR -DED in Fig.3 of Ref. [2]. In Eq. (9) the terms that are proportional to $g_{i k} V^{a, \theta}, g_{i k} B^{a, \theta}$ and their derivatives describe the poloidal modes coupling due to plasma toroidicity in inertial, convective and ponderomotive terms in Eq. (1). In coordinates $\left(\rho_{0}, \omega_{0}\right)$ the solutions of Eqs. (8), (9) take the form

$$
\begin{aligned}
& \left(B^{\rho_{0}}\left(\rho_{0}, \omega_{0}, \zeta, t\right), V^{\rho_{0}}\left(\rho_{0}, \omega_{0}, \zeta, t\right)\right) \\
\approx & \sum_{m, m^{*}}\left\{\begin{array}{c}
B_{m}^{a}\left(\rho_{0}\right) \\
V_{m}^{a}\left(\rho_{0}\right)
\end{array}\right\} J_{m^{*}-m}(m|\lambda|) \exp \left[i\left(m^{*} \omega_{0}-n_{0} \zeta-\omega t\right)\right] .
\end{aligned}
$$

Here $J_{m^{*}-m}(m|\lambda|)$ is the Bessel function. Mode numbers $m^{*}$ and $n_{0}$ are defined by the TEXTOR-DED antenna spectrum $g_{m^{*}}^{(0)}$, this having been investigated in Ref. [4] $\left(m_{0}=20, n_{0}=4\right)$

$$
g_{m^{*}}^{(0)} \propto \frac{\sin \left(m^{*}-m_{0}\right) \theta_{c}}{\pi\left(m^{*}-m_{0}\right)}, \theta_{c}=\pi n_{0} / m_{0} .
$$

The spectrum of perturbations is found from the boundary condition at $\rho_{0}=\rho_{\text {coil }}, \rho_{\text {coil }}$ is the radial position of DED coils. This spectrum is shown in Figure for 
$\beta_{\text {pol }} \approx 1$ and the parabolic profile of the equilibrium current density (compare with $[1,4]), \beta_{p o l}$ is the ratio of the plasma pressure to the pressure of the poloidal magnetic field.

The DED antenna produces the whole spectrum of $m$, but the $m=12$ mode term in Eq.(14) is of specific interest $\left(q\left(a_{\text {res }}\right)=12 / 4\right.$ is the base mode resonance) $[1,4]$

$$
B_{12}^{a}\left(\rho_{0}\right) J_{m^{*}-12}(12|\lambda|), \quad m^{*} \sim 20 .
$$

Due to the small parameter $J_{m^{*}-m}(m|\lambda|) \sim 0.06-0.2$ for $\beta_{p o l} \sim 0.5-1$ the influence of this resonant term strongly drops in comparison with the cylindrical case in Fig. 3 of Ref. [2] (compare with $[1,4])$. Note, that the value of $J_{m^{*}-m}(m|\lambda|)$ strongly depends on the value of $\beta_{p o l}$ and the equilibrium current density profile. Note, that the poloidal driven force drops as $J_{m^{*}-m}^{2}(m|\lambda|)$.

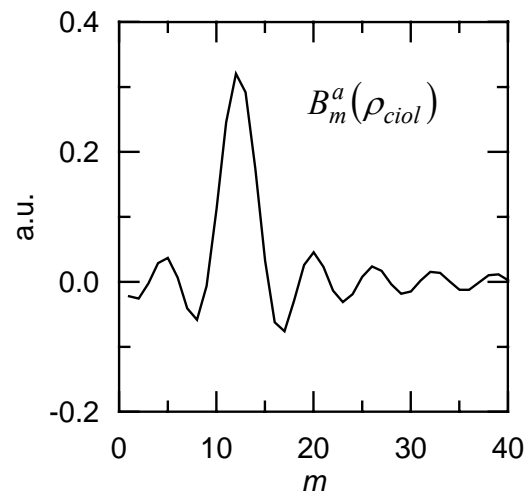

Spectrum of perturbations in Eq. (14)

It was shown in Refs. [1,4], that DED magnetic perturbations (without plasma response being taken into account) decrease with the distance from the DED coils as

$$
B_{r}^{\sim} \propto\left(r / r_{\text {coil }}\right)^{m^{*}-1} .
$$

Our consideration (see Eq.(16)) shows a more complicated behavior of the main resonant term compared with Eq. (17). Moreover, the behavior of the resonant term in our case (Eq.(16)) is not so predictable in the experiment in comparison with Eq.(17).

Estimates show, that for typical TEXTOR-DED parameters the resistive term in Eq. (8) is only two or three times larger than the toroidal coupling term. For $m^{2}>>1$ the last term in Eq. (8) may be the same order of magnitude as the toroidal coupling terms.

\section{CONCLUSIONS}

The equations that describe the penetration of external helical magnetic perturbations have been derived for the toroidal geometry in the first-order of $a / R$ approximation. The role of toroidal effects is estimated.

It is shown that as a first approximation it is possible to use the results of cylindrical model [2] with the small factor $J_{m^{*}-m}(m|\lambda|)$ being taken into account. This factor strongly reduces the effect of DED coil field.

\section{REFERENCES}

1. M.W. Jakubowski, S.S. Abdullaev, K.H. Finken. Modelling of the magnetic field structures and first measurements of heat fluxes for TEXTOR-DED operation//Nucl. Fusion (44). 2004, No.6, p.S1-S11.

2. I.M. Pankratov, A.Ya. Omelchenko, V.V. Olshansky and K.H. Finken. Investigation of plasma response influence on the penetration of an external low frequency helical perturbation into a tokamak edge plasma// Nucl. Fusion (44). 2004, No.6, p.S37-S43.

3. A.B. Mikhailovskii. Instabilities of plasma in magnetic traps. Moscow: "Atomizdat", 1978 (in Russian).

4. S.S Abdullaev, K.H. Finken, K.H. Spatschek. Asymptotical and mapping methods in study of ergodic divertor magnetic field in a toroidal system// Phys. Plasmas (6). 1999, No.1, p.153-174.

\title{
ТОРОИДАЛЬНОЕ РАССМОТРЕНИЕ ОТКЛИКА ПЛАЗМЫ НА ПРОНИКНОВЕНИЕ ВНЕШНЕГО НИЗКОЧАСТОТНОГО ВИНТОВОГО ВОЗМУЩЕНИЯ В КРАЕВУЮ ПЛАЗМУ ТОКАМАКА
}

\author{
И.М. Панкратов, А.Я. Омельченко, В.В. Ольшанский
}

Для адекватного сравнения с экспериментами на токамаке TEXTOR-DED проведено теоретическое исследование влияния поля DED на отклик плазмы в тороидальной геометрии на основе двужидкостной МГД.

\section{ТОРОІДАЛЬНИЙ РОЗГЛЯД ВІДГУКУ ПЛАЗМИ НА ПРОНИКНЕННЯ ЗОВНШШНЬОГО НИЗЬКОЧАСТОТНОГО ГВИНТОВОГО ЗБУРЕННЯ У КРАЙОВУ ПЛАЗМУ ТОКАМАКА}

\section{І.М. Панкратов, А.Я. Омельченко, В.В. Ольщанський}

Для адекватного порівняння 3 експериментами на токамаці TEXTOR-DED проведено теоретичне дослідження впливу поля DED на відгук плазми в тороідальній геометрії на основі дворідинної МГД. 